\title{
Association between Stress-Coping Strategy and Functional Disability in the General Older Adult Population: The Takashima Study
}

\author{
Naoyuki Takashima a,b Yasuyuki Nakamurab, c Naoko Miyagawab, d \\ Aya Kadotab, e Sachiko Tanaka-Mizunob,f Kenji Matsuig ${ }^{\text {batsuyuki Miurab,e }}$ \\ Hirotsugu Ueshimab, e Yoshikuni Kitab,h on behalf of the Takashima Study Group

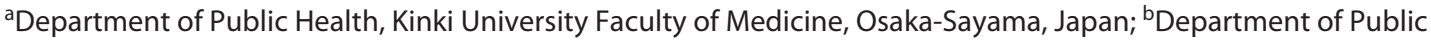 \\ Health, Shiga University of Medical Science, Otsu, Japan; ${ }^{\top}$ Yamashina Racto Clinic and Medical Examination \\ Center, Kyoto, Japan; dDepartment of Preventive Medicine and Public Health, Keio University School of Medicine, \\ Shinjuku-ku, Japan; eNCD Epidemiology Research Center, Shiga University of Medical Science, Otsu, Japan; \\ ${ }^{\mathrm{f}}$ Graduate School of Medicine, Kyoto University, Kyoto, Japan; ${ }^{9}$ Center for Public Health Sciences, National Cancer \\ Center Japan, Chuo-ku, Japan; hFaculty of Nursing Science, Tsuruga Nursing University, Tsuruga, Japan
}

\section{Keywords}

Cohort study - Asian population - Positive thought .

Problem-solving

\begin{abstract}
Background: Both physical and psychological factors have been associated with functional disability. However, the associations between stress-coping strategies and future functional disability remain unclear. Methods: We analyzed 2,924 participants who did not have incidence of functional disability or death within the first 3 years of the baseline survey and were aged 65 years or more at the end of follow-up. Stress-coping strategies were assessed via a self-administered questionnaire (emotional expression, emotional support seeking, positive thought, problemsolving, and disengagement) in a baseline survey from 2006 to 2014. Levels of coping strategies were classified as low, middle, and high based of frequency. Functional disability decline was followed up using the long-term-care
\end{abstract}

insurance program until November 1, 2019. Functional disability decline was defined as a new long-term-care insurance program certification. Cox proportional hazards model with competing risk analysis for death was used to evaluate associations between coping strategy levels and functional disability. Results: During the follow-up period, we observed 341 cases of functional disability and 73 deaths without previous incidence of functional disability. A significant inverse association between "positive thought" and "problem-solving" and future functional disability was observed. Multivariable adjusted hazard ratios (95\% confidence interval) for functional disability were $0.68(0.51-0.92)$ for high levels of "positive thought" and $0.73(0.55-0.95)$ for high levels of "problem-solving," compared with low levels of the coping strategies. The inverse association was stronger in men. Conclusions: Some subcomponents of stress-coping strategies might be associated with future incidence of functional disability among older adults.

(c) 2021 The Author(s).

Published by S. Karger AG, Basel
(C) 2021 The Author(s)

Published by S. Karger AG, Basel

This is an Open Access article licensed under the Creative Commons Attribution-NonCommercial-4.0 International License (CC BY-NC) (http://www.karger.com/Services/OpenAccessLicense), applicable to the online version of the article only. Usage and distribution for commercial purposes requires written permission. 


\section{Introduction}

Japan is currently the most aged society in the world with a reported percentage of the population aged 65 years and over (aging rate) of $28.1 \%$ in 2018 [1]. The average period with no impediment to daily life was estimated to be 72 years in men and 75 years in women, and the average period with impediment to daily life was estimated to be 8.8 years in men and 12.3 years in women in 2016 [1]. The World Population Prospects 2019 reported that the aging rate in developed countries will be $28.2 \%$ in 2060, while the aging rate in East Asia and Southern Europe will exceed 30\% in 2060 [2]. Other developed countries, particularly in East Asia and Europe, will undergo super-aging, indicating that the expansion of healthy life expectancy is becoming an increasingly urgent issue.

Stress-coping strategy has been associated with both chronic disease and psychological health [3]. Approachoriented coping strategies were related to the volume of the hippocampus [4], and the decreased risk of stroke [5] and depression [6]. On the other hand, avoidance coping strategies were associated with increased risk of depression [6]. Both physical status [7] such as smoking [8,9], high blood pressure (BP) [10], and physical frailty [11], and psychological factors, including depression $[7,12]$ were related to future functional decline. A recent study in Japan found that mild or moderate psychological distress was associated with the incidence of functional disability [13]. The major estimated causes of long-term care in Japan were dementia (17.6\%), stroke (16.1\%), and infirmity due to aging (12.8\%) [14]. These results might strongly suggest that stress-coping strategies might be associated with future functional disability via these risk factors. However, to the best of our knowledge, no previous studies have examined the association between (or subcomponents of) stress-coping strategies and future functional disability in the general older adult population. The current study aimed to examine the association between stress-coping strategies and future functional disability in the general Japanese older adult population using the Takashima study, an ongoing population-based cohort study in Japan $[15,16]$.

\section{Participants and Methods}

\section{Participants and Follow-Up}

The Takashima study is an ongoing population-based cohort study of risk factors for lifestyle-related disease $[15,16]$ and is part of the Japan Multi-Institutional Collaborative Cohort (J-MICC) study $[17,18]$. From 2006 to 2014 , a total of 4,760 residents in Ta- kashima City aged 20 years or more who underwent the annual health check-up agreed to participate in the baseline survey.

To minimize reverse causation as much as possible, we excluded participants with a history of cardiovascular disease (CVD) $(N=169)$ and those with missing information $(N=153)$ in the baseline survey. We further excluded participants aged $<65$ years at the end of follow-up $(N=1,420)$, those who died or exhibited incident functional disability within the first 3 years of follow-up $(N=78)$, and those who were lost to follow-up $(N=16)$ due to incomplete data. Consequently, 2,924 participants (1,195 men and 1,729 women) were included in the current analysis.

The Takashima study conducted follow-up surveys until November 1, 2019. Vital status and functional disability of the participants were determined from the basic resident registry and long-term-care insurance program of the local government. The long-term-care insurance program is a public social health program providing long-term care in Japan $[19,20]$. An individual aged 65 years or more is eligible for long-term care. To receive long-term-care services through the long-term-care insurance program, the individual is assessed for functional disability using the standardized questionnaire developed by the Ministry of Health, Labour, and Welfare of Japan. If the individual is eligible for care, the committee decides the level of care, ranging from $\mathrm{Re}$ quiring-support 1, Requiring-support 2, Care level 1, to Care level 5 according to the national standardized criteria [21]. During the follow-up period, the criteria for the long-term-care program were not changed. Functional disability decline in the current study was defined as a new long-term-care insurance program certification of requiring support (Requiring-support 1 and higher) in accordance with the methods used in the previous studies [13, 22, 23]. Several previous studies have used long-term-care insurance program certification as an outcome of incidence of functional disability in older adults [13, 22-27], and 1 previous study reported a correlation between long-term-care insurance program certification, activities of daily living, and Mini-Mental State Examination scores [28].

\section{Baseline Examinations}

Baseline BP was measured twice by trained observers using a standard electrical sphygmomanometer BP103iII (Omron Healthcare, Kyoto, China) applied to the right arm of seated participants after at least $5 \mathrm{~min}$ of rest. Body mass index (BMI) was calculated as weight divided by height squared $\left(\mathrm{kg} / \mathrm{m}^{2}\right)$. We used a self-administered questionnaire that included lifestyle, clinical history, family history, smoking, and alcohol drinking habits. The questionnaire was checked and collected by trained observers.

Nonfasting blood samples were obtained at the baseline survey. Blood samples were shipped to 1 laboratory (Kinkiyoken, Otsu, Japan) for blood measurements. Hemoglobin A1c (HbA1c) level (JDS) was measured by latex agglutination immunoassay. HbA1c (NGSP) levels were calculated using a formula $(1.02 \times \mathrm{HbA} 1 \mathrm{c}$ [JDS] + 0.25) [29]. Serum triglyceride, low-density lipoprotein cholesterol, and high-density lipoprotein cholesterol levels were measured by direct enzymatic assays (Sekisui Medical Co., Ltd., Tokyo, Japan) using standardized protocol certification by the Cholesterol Reference Method Laboratory Network.

\section{Coping Strategies}

Coping strategies at the baseline survey were assessed using a self-administered questionnaire. The questionnaire to assess 
stress-coping strategies used by the J-MICC study has been described elsewhere [30]. Briefly, participants were asked "How do you cope with daily life?" and requested to report the frequency (seldom, occasionally, often, and very often) of using 5 coping strategies (emotional expression, emotional support seeking, positive thought, problem-solving, and disengagement) selected from the Brief COPE [31] or the General Coping Questionnaire [32]. The 5 coping strategies were evaluated with the following items: (1) "To express your feeling or your undesirable emotion" (emotional expression); (2) "To consult someone close to you and to ask for encouragement" (emotional support seeking); (3) "To interpret that problem positively" (positive thought); (4) "To work hard to resolve the problem" (problem-solving); and (5) "To let the problem take its own course" (disengagement).

\section{Statistical Analysis}

Cox proportional hazards models with competing risk analysis for death [33] were used to estimate adjusted hazard ratios (HRs) and $95 \%$ confidence intervals (CIs) of the incidence of functional disability based on the levels of each stress-coping strategy with low level as a reference. The level of each coping strategy was classified as follows: low for a frequency of "seldom," middle for a frequency of "occasionally," and high for a frequency of "often" or "very often." Age- and sex-adjusted HRs for the incidence of functional disability were estimated using models adjusted for age and sex (model 1). Multivariable-adjusted HRs for the incidence of functional disability were estimated using models adjusted for age, sex, systolic BP, BMI, HbA1c (NGSP) level, low-density lipoprotein cholesterol level, high-density lipoprotein cholesterol level, drinking habits (nondrinker, past-drinker, or current drinker), smoking habits (nonsmoker, past-smoker, or current smoker), antihypertensive medication, and medication for dyslipidemia and diabetes (model 2). We added the number of years of education as a covariate in an additional model (model 3). Similarly, we conducted a subgroup analysis by sex. All tests were 2-tailed, and $p$ values of $<0.05$ were considered to indicate statistical significance. All analyses were performed using SAS 9.4 (SAS Institute, Cary, NC, USA).

\section{Results}

The total person-years of observation were 27,270 , and the mean follow-up period was 9.33 years. Participants' baseline characteristics are shown in Table 1. The mean age was 66.3 years, and mean systolic $\mathrm{BP}$ and diastolic $\mathrm{BP}$ were $129.6 \mathrm{~mm} \mathrm{Hg}$ and $76.3 \mathrm{~mm} \mathrm{Hg}$, respectively. The mean number of years of education was 11.2 years. During the follow-up period, 366 participants had functional disability, and 73 participants died without incidence of functional disability.

Table 2 shows adjusted HRs (95\% CI) for all participants based on the level of stress-coping strategies. Compared with low levels for the coping strategy, the multivariable-adjusted HRs for functional disability with high levels of "positive thought" and "problem-solving" were 0.68 (95\% CI, 0.51, 0.92) and 0.73 (95\% CI, 0.55, 0.95),
Table 1. Baseline characteristics of study population: the Takashima Study, Japan, 2006-2014

\begin{tabular}{lll}
\hline Participants & & \\
\hline Total number & 2,924 & \\
Age, years, mean (SD) & 66.27 & $(5.57)$ \\
Men, $N$ (\%) & 1,195 & $(40.87)$ \\
Systolic BP, mm Hg, mean (SD) & 129.57 & $(19.54)$ \\
Diastolic BP, mm Hg, mean (SD) & 76.31 & $(11.20)$ \\
BMI, kg/m², mean (SD) & 23.07 & $(2.99)$ \\
HbA1c, NGSP, \%, mean (SD) & 5.61 & $(0.65)$ \\
LDL cholesterol, mmol/L, mean (SD) & 3.32 & $(0.83)$ \\
HDL cholesterol, mmol/L, mean (SD) & 1.60 & $(0.40)$ \\
Education year, year, mean (SD) & 11.13 & $(2.21)$ \\
Drinking status & & \\
$\quad$ Nondrinker, $N$ (\%) & 1,541 & $(52.70)$ \\
$\quad$ Past-drinker, $N(\%)$ & 58 & $(1.98)$ \\
$\quad$ Current drinker, $N$ (\%) & 1,325 & $(45.31)$ \\
Smoking status & & \\
$\quad$ Nonsmoker, $N(\%)$ & 2,106 & $(72.02)$ \\
$\quad$ Past-smoker, $N(\%)$ & 471 & $(16.11)$ \\
$\quad$ Current smoker, $N$ (\%) & 347 & $(11.87)$ \\
Medication & & \\
$\quad$ Hypertension, $N(\%)$ & 882 & $(30.16)$ \\
$\quad$ Dyslipidemia, $N$ (\%) & 510 & $(17.44)$ \\
$\quad$ Diabetes, $N$ (\%) & 180 & $(6.16)$ \\
\hline
\end{tabular}

$N$, number; LDL, low-density lipoprotein; HDL, high-density lipoprotein.

respectively. The significant associations between future functional disability and these coping strategies remained after additional adjustment for the number of years of education. However, other coping strategies were not associated with future functional disability.

Adjusted HRs (95\% CI) based on the level of coping strategies stratified by sex are shown in Table 3 for men and Table 4 for women. High levels of both "positive thought" and "problem-solving" were significantly associated with increased risk of future functional disability in men. Compared with low levels of coping strategies, the multivariable-adjusted HRs of future functional disability in men were 0.61 (95\% CI, 0.41, 0.92) for high levels of "positive thought" and 0.63 (95\% CI: $0.43,0.94)$ for high levels of "problem-solving." In women, the multivariable-adjusted HRs were 0.72 (95\% CI: $0.48,1.09)$ for high levels of "positive thought" and 0.81 (95\% CI: 0.55, 1.21) for high levels of "problem-solving." Similar results were observed in both men and women, but no reach statistical significance was noted in women. Other coping strategies in both men and women were not associated with future functional disability. 
Table 2. HRs for functional disability based on the levels of coping strategies in all participants: the Takashima Study, Japan, 2006-2014

\begin{tabular}{|c|c|c|c|c|c|c|}
\hline & \multicolumn{6}{|c|}{ Level of coping strategies } \\
\hline & low & middle & & high & & $p$ trend \\
\hline \multicolumn{7}{|c|}{ Emotional expression } \\
\hline Person-years & 8,120 & 15,351 & & 3,799 & & \\
\hline Events, $N$ & 143 & 173 & & 50 & & \\
\hline Model 1 & 1.00 (reference) & 0.97 & $(0.77-1.22)$ & 1.13 & $(0.82-1.55)$ & 0.635 \\
\hline Model 2 & 1.00 (reference) & 0.99 & $(0.78-1.24)$ & 1.19 & $(0.87-1.63)$ & 0.459 \\
\hline Model 3 & 1.00 (reference) & 0.99 & $(0.78-1.25)$ & 1.20 & $(0.87-1.64)$ & 0.429 \\
\hline \multicolumn{7}{|c|}{ Emotional support seeking } \\
\hline Person-years & 12,305 & 10,901 & & 4,064 & & \\
\hline Events, $N$ & 186 & 137 & & 43 & & \\
\hline Model 1 & 1.00 (reference) & 1.03 & $(0.82-1.29)$ & 0.97 & $(0.69-1.37)$ & 0.979 \\
\hline Model 2 & 1.00 (reference) & 1.09 & $(0.87-1.38)$ & 0.96 & $(0.68-1.37)$ & 0.895 \\
\hline Model 3 & 1.00 (reference) & 1.09 & $(0.87-1.37)$ & 0.96 & $(0.67-1.36)$ & 0.910 \\
\hline \multicolumn{7}{|l|}{ Positive thought } \\
\hline Person-years & 3,180 & 9,989 & & 14,101 & & \\
\hline Events, $N$ & 67 & 132 & & 167 & & \\
\hline Model 1 & 1.00 (reference) & 0.82 & $(0.61-1.11)$ & 0.67 & $(0.50-0.89)$ & 0.004 \\
\hline Model 2 & 1.00 (reference) & 0.85 & $(0.62-1.15)$ & 0.68 & $(0.51-0.92)$ & 0.007 \\
\hline Model 3 & 1.00 (reference) & 0.85 & $(0.62-1.15)$ & 0.69 & $(0.51-0.93)$ & 0.009 \\
\hline \multicolumn{7}{|l|}{ Problem-solving } \\
\hline Person-years & 3,826 & 9,406 & & 14,038 & & \\
\hline Events, $N$ & 88 & 110 & & 168 & & \\
\hline Model 1 & 1.00 (reference) & 0.81 & $(0.60-1.09)$ & 0.74 & $(0.56-0.96)$ & 0.031 \\
\hline Model 2 & 1.00 (reference) & 0.78 & $(0.57-1.06)$ & 0.73 & $(0.55-0.95)$ & 0.030 \\
\hline Model 3 & 1.00 (reference) & 0.78 & $(0.58-1.06)$ & 0.73 & $(0.56-0.96)$ & 0.034 \\
\hline \multicolumn{7}{|l|}{ Disengagement } \\
\hline Person-years & 5,647 & 12,299 & & 9,324 & & \\
\hline Events, $N$ & 79 & 159 & & 128 & & \\
\hline Model 1 & 1.00 (reference) & 1.06 & $(0.81-1.4)$ & 1.15 & $(0.86-1.52)$ & 0.339 \\
\hline Model 2 & 1.00 (reference) & 1.11 & $(0.85-1.47)$ & 1.18 & $(0.88-1.58)$ & 0.264 \\
\hline Model 3 & 1.00 (reference) & 1.12 & $(0.85-1.47)$ & 1.19 & $(0.89-1.59)$ & 0.252 \\
\hline
\end{tabular}

Model 1 was adjusted for age and sex. Model 2 was adjusted for age, sex, systolic BP, body mass index, HbA1c(NGSP), LDL cholesterol, HDL cholesterol, drinking habits, smoking habits, antihypertensive medication, medication for dyslipidemia, and medication for diabetes. Model 3 was adjusted for covariates in model 2 and number of years of education. BP, blood pressure; HbA1c, hemoglobin A1c; LDL, low-density lipoprotein; HDL, highdensity lipoprotein; $H R$, hazard ratio.

\section{Discussion}

In the present study, we found that several components of stress-coping strategies were associated with future functional disability defined as long-term-care insurance program certification. The levels of "positive thought" and "problem solving" were inversely associated with future functional disability in the general Japanese older adult population. The associations were more prominent in men. However, no significant association between future functional disability and "emotional expression," "emotional support seeking," and "disengagement" were observed in this study.
Previous studies reported an association between functional disability and lifestyle-related factors, psychological health, and physical health $[7,11,12]$. Coping strategies were associated with lifestyle, psychological health, and physical health $[3,5,6,30,34]$. These studies suggested that coping strategies might be associated with functional disability through these factors. However, to the best of our knowledge, there is little evidence regarding the association between coping strategies and future functional disability, and this is the first study to examine the association between components of coping strategies and future functional disability using a population-based cohort study. 
Table 3. HRs for functional disability based on the levels of coping strategies in men: the Takashima Study, Japan, 2006-2014

\begin{tabular}{|c|c|c|c|c|c|c|}
\hline & \multicolumn{6}{|c|}{ Level of coping strategies } \\
\hline & low & middle & & high & & $p$ trend \\
\hline \multicolumn{7}{|c|}{ Emotional expression } \\
\hline Person-years & 3,292 & 6,355 & & 1,521 & & \\
\hline Events, $N$ & 56 & 88 & & 20 & & \\
\hline Model 1 & 1.00 (reference) & 1.09 & $(0.77-1.54)$ & 1.16 & $(0.71-1.89)$ & 0.517 \\
\hline Model 2 & 1.00 (reference) & 1.12 & $(0.79-1.59)$ & 1.19 & $(0.72-1.96)$ & 0.436 \\
\hline Model 3 & 1.00 (reference) & 1.14 & $(0.80-1.61)$ & 1.21 & $(0.74-2.00)$ & 0.375 \\
\hline \multicolumn{7}{|c|}{ Emotional support seeking } \\
\hline Person-years & 7,218 & 3,160 & & 791 & & \\
\hline Events, $N$ & 113 & 43 & & 8 & & \\
\hline Model 1 & 1.00 (reference) & 0.98 & $(0.69-1.39)$ & 0.73 & $(0.35-1.56)$ & 0.506 \\
\hline Model 2 & 1.00 (reference) & 0.95 & $(0.66-1.36)$ & 0.78 & $(0.36-1.70)$ & 0.527 \\
\hline Model 3 & 1.00 (reference) & 0.92 & $(0.64-1.32)$ & 0.76 & $(0.35-1.66)$ & 0.441 \\
\hline \multicolumn{7}{|l|}{ Positive thought } \\
\hline Person-years & 1,530 & 4,018 & & 5,620 & & \\
\hline Events, $N$ & 38 & 54 & & 72 & & \\
\hline Model 1 & 1.00 (reference) & 0.77 & $(0.50-1.18)$ & 0.61 & $(0.40-0.91)$ & 0.014 \\
\hline Model 2 & 1.00 (reference) & 0.78 & $(0.51-1.19)$ & 0.61 & $(0.41-0.92)$ & 0.018 \\
\hline Model 3 & 1.00 (reference) & 0.78 & $(0.51-1.20)$ & 0.63 & $(0.41-0.95)$ & 0.025 \\
\hline \multicolumn{7}{|l|}{ Problem-solving } \\
\hline Person-years & 1,618 & 3,396 & & 6,154 & & \\
\hline Events, $N$ & 45 & 40 & & 79 & & \\
\hline Model 1 & 1.00 (reference) & 0.70 & $(0.45-1.11)$ & 0.67 & $(0.45-0.98)$ & 0.056 \\
\hline Model 2 & 1.00 (reference) & 0.64 & $(0.40-1.02)$ & 0.63 & $(0.43-0.94)$ & 0.046 \\
\hline Model 3 & 1.00 (reference) & 0.64 & $(0.40-1.03)$ & 0.65 & $(0.44-0.97)$ & 0.064 \\
\hline \multicolumn{7}{|l|}{ Disengagement } \\
\hline Person-years & 2,904 & 5,062 & & 3,202 & & \\
\hline Events, $N$ & 45 & 71 & & 48 & & \\
\hline Model 1 & 1.00 (reference) & 1.01 & $(0.69-1.47)$ & 1.05 & $(0.7-1.57)$ & 0.831 \\
\hline Model 2 & 1.00 (reference) & 1.05 & $(0.72-1.55)$ & 1.14 & $(0.74-1.74)$ & 0.551 \\
\hline Model 3 & 1.00 (reference) & 1.05 & $(0.72-1.55)$ & 1.15 & $(0.75-1.76)$ & 0.519 \\
\hline
\end{tabular}

Model 1 was adjusted for age. Model 2 was adjusted for age, systolic BP, body mass index, HbA1c(NGSP), LDL cholesterol, HDL cholesterol, drinking habits, smoking habits, antihypertensive medication, medication for dyslipidemia, and medication for diabetes. Model 3 was adjusted for covariates in model 2 and the number of years of education. BP, blood pressure; HbA1c, hemoglobin A1c; LDL, low-density lipoprotein; HDL, high-density lipoprotein; HR, hazard ratio.

In the current study, high levels of "positive thought" and "problem-solving" were significantly associated with a lower risk of future functional disability. Previous studies have shown that problem-solving and positive reappraisal strategies are inversely associated with depression [6] and mental health [35]. Other studies have revealed that the approach-oriented coping strategies are positively associated with the volume of the whole hippocampus [4]. Moreover, depression, mental health, and cognitive impairment are reported to be risk factors of functional disability [12]. The current results are consistent with these previous findings. The preventive effects of coping strategies for depression, mental health, and cognitive impairment may be mechanisms associated with coping strategies and future functional disability.

In Japan, a previous study reported that dementia, stroke, and frailty due to aging were the main causes of functional disability decline, defined as long-term-care insurance program certification of requiring any support, which was the same definition of functional disability used in the current study [14]. In the Japan Public Health Center cohort study, stroke incidence was inversely associated with the approach-oriented coping strategy [5]. These previous findings were also consistent with the cur- 
Table 4. HRs for functional disability based on the levels of coping strategies in women: the Takashima Study, Japan, 2006-2014

\begin{tabular}{|c|c|c|c|c|c|c|}
\hline & \multicolumn{6}{|c|}{ Level of coping strategies } \\
\hline & low & middle & & high & & $p$ trend \\
\hline \multicolumn{7}{|c|}{ Emotional expression } \\
\hline Person-years & 4,828 & 8,995 & & 2,279 & & \\
\hline Events, $N$ & 87 & 85 & & 30 & & \\
\hline Model 1 & 1.00 (reference) & 0.89 & $(0.65-1.22)$ & 1.11 & $(0.73-1.68)$ & 0.911 \\
\hline Model 2 & 1.00 (reference) & 0.88 & $(0.65-1.20)$ & 1.09 & $(0.71-1.67)$ & 0.977 \\
\hline Model 3 & 1.00 (reference) & 0.88 & $(0.64-1.20)$ & 1.08 & $(0.71-1.66)$ & 0.991 \\
\hline \multicolumn{7}{|c|}{ Emotional support seeking } \\
\hline Person-years & 5,087 & 7,742 & & 3,274 & & \\
\hline Events, $N$ & 73 & 94 & & 35 & & \\
\hline Model 1 & 1.00 (reference) & 1.08 & $(0.79-1.48)$ & 1.09 & $(0.73-1.64)$ & 0.612 \\
\hline Model 2 & 1.00 (reference) & 1.20 & $(0.88-1.63)$ & 1.08 & $(0.71-1.65)$ & 0.543 \\
\hline Model 3 & 1.00 (reference) & 1.19 & $(0.87-1.63)$ & 1.08 & $(0.70-1.65)$ & 0.549 \\
\hline \multicolumn{7}{|l|}{ Positive thought } \\
\hline Person-years & 1,649 & 5,972 & & 8,481 & & \\
\hline Events, $N$ & 29 & 78 & & 95 & & \\
\hline Model 1 & 1.00 (reference) & 0.86 & $(0.55-1.33)$ & 0.71 & $(0.47-1.09)$ & 0.088 \\
\hline Model 2 & 1.00 (reference) & 0.89 & $(0.58-1.37)$ & 0.72 & $(0.48-1.09)$ & 0.077 \\
\hline Model 3 & 1.00 (reference) & 0.88 & $(0.57-1.36)$ & 0.72 & $(0.47-1.09)$ & 0.073 \\
\hline \multicolumn{7}{|l|}{ Problem-solving } \\
\hline Person-years & 2,208 & 6,010 & & 7,884 & & \\
\hline Events, $N$ & 43 & 70 & & 89 & & \\
\hline Model 1 & 1.00 (reference) & 0.89 & $(0.60-1.33)$ & 0.79 & $(0.54-1.16)$ & 0.211 \\
\hline Model 2 & 1.00 (reference) & 0.92 & $(0.61-1.38)$ & 0.81 & $(0.55-1.21)$ & 0.274 \\
\hline Model 3 & 1.00 (reference) & 0.91 & $(0.61-1.37)$ & 0.81 & $(0.55-1.19)$ & 0.255 \\
\hline \multicolumn{7}{|l|}{ Disengagement } \\
\hline Person-years & 2,743 & 7,237 & & 6,122 & & \\
\hline Events, $N$ & 34 & 88 & & 80 & & \\
\hline Model 1 & 1.00 (reference) & 1.14 & $(0.76-1.73)$ & 1.28 & $(0.84-1.93)$ & 0.231 \\
\hline Model 2 & 1.00 (reference) & 1.22 & $(0.81-1.84)$ & 1.34 & $(0.88-2.03)$ & 0.180 \\
\hline Model 3 & 1.00 (reference) & 1.22 & $(0.81-1.83)$ & 1.33 & $(0.88-2.02)$ & 0.183 \\
\hline
\end{tabular}

Model 1 was adjusted for age. Model 2 was adjusted for age, systolic BP, BMI, HbA1c(NGSP), LDL cholesterol, HDL cholesterol, drinking habits, smoking habits, antihypertensive medication, medication for dyslipidemia, and medication for diabetes. Model 3 was adjusted for covariates in model 2 and number of years of education. BP, blood pressure; BMI, body mass index; HbA1c, hemoglobin A1c; LDL, low-density lipoprotein; HDL, high-density lipoprotein; HR, hazard ratio.

rent results, and the preventive effect of approach-oriented coping strategies for stroke might also provide a potential mechanism underlying our observations.

In the present study, we could not find any significant association between emotional expression, emotional support seeking, or avoidance-oriented behaviors. A previous study showed to be no significant association between BMI and emotional expression or emotional support seeking [30]. Furthermore, depression was not associated with emotional support seeking [6]. Avoidance-oriented behaviors were successful for coping with short-term uncontrollable stress and related to chronic disease progression or mortality [3]. Another study from Japan reported that avoidance-oriented behaviors were not associated with CVD-related death or incidence [5]. These results suggested that these coping strategies might not be effective for coping with long-term stress.

Significant inverse associations between "positive thought" and "problem-solving" and future functional disability were found in men, but in women, a similar but not significant association was found. Sex differences in the association between these coping strategies and Creactive protein (CRP) level [34] were reported to be observed. These coping strategies were associated with CRP 
level in men. CRP is a predictor for future CVD-related death [36]. Another study reported that these coping strategies resulted in lower risk of depression in both men and women, but the adjusted odds ratios for depression in men was 0.52 , which was lower than that in women (0.64) [6]. The previous studies suggested that these coping strategies might be more strongly influenced by both physical and psychological factors that could be considered potential risk factors for functional disability in men compared with women.

The strengths of the current study include its prospective design and complete follow-up of functional disability using the long-term-care insurance program of the local government. We excluded men and women who had incident functional disability or died within the first 3 years of the baseline survey from the analysis to minimize bias due to reverse causation. Follow-up of functional disability using the long-term-care insurance program might minimize bias due to loss to follow-up bias. However, the present study involved several limitations that should be considered. First, we did not consider income in our analysis. Education and income are considered major components of socioeconomic status. In the current study, we considered only education, and the association between components of stress-coping strategies and functional disability was not significantly affected by the education level. Second, we did not consider changes in coping strategies during the follow-up period. Third, due to a lack of data, we did not consider depression and dementia at baseline as potential confounders. Lastly, due to insufficient data, we did not consider the presence or absence of a family member living with the participants.

\section{Conclusion}

The present study demonstrated that some subcomponents of coping strategies ("positive thought" and "problem-solving") are independent predictors of future functional disability defined as long-term-care insurance program certification in the general Japanese older adult population. These findings potentially indicate that improved coping strategies may prevent future functional disability.

\section{Acknowledgments}

We thank the members of the Takashima study for their contribution. We thank Enago (Crimson Interactive Japan Co., Ltd. Tokyo, Japan) for editing the draft of the manuscript.

\section{Statement of Ethics}

This study protocol adhered to the Declaration of Helsinki and the ethical standards of the responsible committee on human experimentation. All participants provided written informed consent, and this study protocol was approved by the Institutional Review Board of Tsuruga Nursing University (No. 19002), Shiga University of Medical Science (No. G2005-103), and Kinki University Faculty of Medicine (No. 31-165).

\section{Conflict of Interest Statement}

The Takashima study was partially supported by Omron Healthcare Co., Ltd. No other author has reported a potential conflict of interest relevant to this article.

\section{Funding Sources}

This study was supported by the Ministry of Health, Labour, and Welfare, Japan (grants from Research on Cardiovascular Disease [3A-1, 6A-5, and 7A-2] and Comprehensive Research on Cardiovascular and Lifestyle-Related Diseases [H18-CVDIppan-029]); Japan Arteriosclerosis Prevention Fund; Omron Healthcare Co., Ltd; and the Ministry of Education, Culture, Sports, Science, and Technology, Japan, and the Japan Society for the Promotion of Science (the Grants-in-Aid for Scientific Research [13670361, 17390186, 20390184, 20790424, 22790544, $24390165,16 \mathrm{H} 06277,17 \mathrm{H} 04133,19 \mathrm{H} 03902$, and 20K10484] and the Grant-in-Aid for Scientific Research on Priority Areas of Cancer [No. 17015018] and Innovative Areas [No. 221S0001]).

\section{Author Contributions}

Kita Y. and Ueshima H. contributed to the design of Takashima Cohort Study, and Takashima N. and Kita Y. contributed to the conception and design of this study. Takashima N., Nakamura Y., Miyagawa N., Kadota A., Tanaka-Mizuno S., Matsui K., Miura K., Ueshima H., and Kita Y. collected the data. Takashima N. analyzed data and wrote the manuscript draft. All authors discussed the results and contributed to the final manuscript. All authors have approved the submitted manuscript. The manuscript has not been submitted elsewhere nor published elsewhere in whole.

\section{Data Availability Statement}

The data that support the findings of this study are not publicly available due to their containing information that could compromise research participant privacy but are available from the corresponding author (N.T.) upon reasonable request within the limitations of informed consent by the research committee of Takashima Study upon acceptance.
Stress-Coping Strategy and Future

Functional Disability
Gerontology 2022;68:699-706

DOI: $10.1159 / 000519194$ 


\section{References}

1 Japan Cabinet Office. Annual report on the Aging Society; 2019. https://www8.cao.go.jp/ kourei/whitepaper/w-2019/html/zenbun/index.html.

2 World population Prospects 2019. 2019 [cited 2020 Oct 6]; Available from: https://population.un.org/wpp/.

3 Taylor SE, Stanton AL. Coping resources, coping processes, and mental health. Annu Rev Clin Psychol. 2007;3:377-401.

4 Kida H, Nakajima S, Shikimoto R, Ochi R, Noda Y, Tsugawa S, et al. Approach-oriented coping strategy level may be related to volume of the whole hippocampus in the elderly. Psychiatry Clin Neurosci. 2020;74(4):270-6.

5 Svensson T, Inoue M, Sawada N, Yamagishi $\mathrm{K}$, Charvat H, Saito I, et al. Coping strategies and risk of cardiovascular disease incidence and mortality: the Japan public health centerbased prospective study. Eur Heart J. 2016; 37(11):890-9.

6 Nagase Y, Uchiyama M, Kaneita Y, Li L, Kaji $\mathrm{T}$, Takahashi S, et al. Coping strategies and their correlates with depression in the Japanese general population. Psychiatry Res. 2009; 168(1):57-66.

7 Tas U, Verhagen AP, Bierma-Zeinstra SM, Odding E, Koes BW. Prognostic factors of disability in older people: a systematic review. Br J Gen Pract. 2007;57(537):319-23.

8 Takashima N, Miura K, Hozawa A, Okamura $\mathrm{T}$, Hayakawa T, Okuda N, et al. Cigarette smoking in middle age and a long-term risk of impaired activities of daily living: NIPPON DATA80. Nicotine Tob Res. 2010;12(9):9449.

9 Rist PM, Capistrant BD, Wu Q, Marden JR, Glymour MM. Dementia and dependence: do modifiable risk factors delay disability? Neurology. 2014;82(17):1543-50.

10 Hozawa A, Okamura T, Murakami Y, Kadowaki T, Okuda N, Takashima N, et al. High blood pressure in middle age is associated with a future decline in activities of daily living. NIPPON DATA80. J Hum Hypertens. 2009;23(8):546-52.

11 Vermeulen J, Neyens JC, van Rossum E, Spreeuwenberg MD, de Witte LP. Predicting ADL disability in community-dwelling elderly people using physical frailty indicators: a systematic review. BMC Geriatr. 2011;11:33.

12 Stuck AE, Walthert JM, Nikolaus T, Büla CJ, Hohmann C, Beck JC. Risk factors for functional status decline in community-living elderly people: a systematic literature review. Soc Sci Med. 1999;48(4):445-69.

13 Tomata Y, Watanabe T, Tanji F, Zhang S, Sugawara Y, Tsuji I. The impact of psychological distress on incident functional disability in elderly Japanese: the Ohsaki Cohort 2006 study. Int J Environ Res Public Health. 2018;15(11):2502.
14 Ministry of Health, Labour and Welfare. The results of comprehensive survey of living conditions; 2019. Available from: https://www. mhlw.go.jp/toukei/saikin/hw/k-tyosa/ k-tyosa19/index.html.

15 Takashima N, Turin TC, Matsui K, Rumana N, Nakamura Y, Kadota A, et al. The relationship of brachial-ankle pulse wave velocity to future cardiovascular disease events in the general Japanese population: the Takashima Study. J Hum Hypertens. 2014;28(5):323-7.

16 Turin TC, Kita Y, Rumana N, Takashima N, Kadota A, Matsui K, et al. Brachial-ankle pulse wave velocity predicts all-cause mortality in the general population: findings from the Takashima study. Japan Hypertens Res. 2010;33(9):922-5.

17 Hamajima N; J-MICC Study Group. The Japan multi-institutional Collaborative Cohort Study (J-MICC Study) to detect gene-environment interactions for cancer. Asian Pac Cancer Prev. 2007;8(2):317-23.

18 Wakai K, Hamajima N, Okada R, Naito M, Morita E, Hishida A, et al. Profile of participants and genotype distributions of 108 polymorphisms in a cross-sectional study of associations of genotypes with lifestyle and clinical factors: a project in the Japan multi-institutional collaborative cohort (JMICC) study. J Epidemiol. 2011;21(3):22335.

19 Ikegami N. Public long-term care insurance in Japan. JAMA. 1997;278(16):1310-4.

20 Tsutsui T, Muramatsu N. Care-needs certification in the long-term care insurance system of Japan. J Am Geriatr Soc. 2005;53(3):522-7.

21 Ministry of Health Ministry of Health, Labour and Welfare. Guideline for certification Committee of Needed Long-Term Care 2009; 2009. Available from: https://www.mhlw. go.jp/file/06-Seisakujouhou-12300000Roukenkyoku/0000116033.pdf.

22 Hozawa A, Sugawara Y, Tomata Y, Kakizaki M, Tsuboya T, Ohmori-Matsuda K, et al. Relationship between serum isoflavone levels and disability-free survival among community-dwelling elderly individuals: nested casecontrol study of the Tsurugaya project. J Gerontol A Biol Sci Med Sci. 2013;68(4):46572.

23 Zhang S, Tomata Y, Newson RB, Sugawara Y, Tsuji I. Combined healthy lifestyle behaviours and incident disability in an elderly population: the Ohsaki Cohort 2006 Study. J Epidemiol Community Health. 2018;72(8):679-84.

24 Yamada M, Arai H. Predictive value of frailty scores for healthy life expectancy in community-dwelling older Japanese adults. J Am Med Dir Assoc. 2015;16(11):1002-11.
25 Makizako H, Shimada H, Doi T, Tsutsumimoto K, Suzuki T. Impact of physical frailty on disability in community-dwelling older adults: a prospective cohort study. BMJ Open. 2015;5(9):e008462.

26 Ukawa S, Tamakoshi A, Okada Y, Ito YM, Taniguchi R, Tani Y, et al. Social participation patterns and the incidence of functional disability: the Japan gerontological evaluation study. Geriatr Gerontol Int. 2020;20(8):76572.

27 Tamada Y, Takeuchi K, Yamaguchi C, Saito M, Ohira T, Shirai K, et al. Does laughter predict onset of functional disability and mortality among older Japanese adults? The JAGES prospective cohort study. J Epidemiol. 2021; 31(5):301-7.

28 Arai Y, Zarit SH, Kumamoto K, Takeda A. Are there inequities in the assessment of dementia under Japan's LTC insurance system? Int J Geriatr Psychiatry. 2003;18(4):346-52.

29 Kashiwagi A, Kasuga M, Araki E, Oka Y, Hanafusa $\mathrm{T}$, Ito $\mathrm{H}$, et al. International clinical harmonization of glycated hemoglobin in Japan: from japan diabetes society to national glycohemoglobin standardization program values. J Diabetes Investig. 2012;3(1):39-40.

30 Shimanoe C, Hara M, Nishida Y, Nanri H, Otsuka Y, Nakamura K, et al. Perceived stress and coping strategies in relation to body mass index: cross-sectional study of 12,045 Japanese men and women. PLoS One. 2015;10(2): e0118105.

31 Carver CS. You want to measure coping but your protocol's too long: consider the brief COPE. Int J Behav Med. 1997;4(1):92-100.

32 Sasaki M, Yamasaki K. (Development of a dispositional version of the general coping questionnaire [GCQ] and examination of its reliability and validity). Nihon Koshu Eisei Zasshi. 2002;49(5):399-408.

33 Fine JP, Gray RJ. A proportional hazards model for the subdistribution of a competing risk. J Am Stat Assoc. 1999;94(446):496-509.

34 Shimanoe C, Otsuka Y, Hara M, Nanri H, Nishida Y, Nakamura K, et al. Gender-specific associations of perceived stress and coping strategies with C-reactive protein in middleaged and older men and women. Int J Behav Med. 2014;21(5):821-32.

35 Nowlan JS, Wuthrich VM, Rapee RM. Positive reappraisal in older adults: a systematic literature review. Aging Ment Health. 2015; 19(6):475-84.

36 Ni P, Yu M, Zhang R, Cheng C, He M, Wang $\mathrm{H}$, et al. Dose-response association between $\mathrm{C}$-reactive protein and risk of all-cause and cause-specific mortality: a systematic review and meta-analysis of cohort studies. Ann Epidemiol. 2020;51:20-7.e11. 